\title{
Zero-Bias Anomaly in Magnetic Tunnel Junctions
}

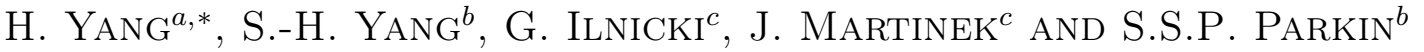 \\ ${ }^{a}$ Department of Electrical and Computer Engineering, National University of Singapore, Singapore \\ ${ }^{b}$ IBM Almaden Research Center, 650 Harry Road, San Jose, California 95120, USA \\ ${ }^{c}$ Institute of Molecular Physics, Polish Academy of Sciences, M. Smoluchowskiego 17, 60-179 Poznań, Poland

\begin{abstract}
We present experimental results, which may indicate the possibility of the coexistence of the Kondo effect and ferromagnetism in macroscopic planar magnetic tunnel junctions with a layer of nanodots inside tunnel barriers. A conductance double peak structure was observed. Magnetic field dependence of the splitting of a conductance peak, and temperature evolution of the conductance curves are well explained from the theoretical point of view according to the predictions of the Kondo physics and cotunneling in the Anderson quantum dot coupled to ferromagnetic leads.
\end{abstract}

PACS numbers: 73.21.La, 73.23.-b, 73.22.-f

\section{Introduction}

Zero-bias anomaly (ZBA) — a departure from smooth conductance background near zero-bias voltage - has been observed widely in various experiments with tunnel junctions in which, deliberately or not, paramagnetic impurities were included [1,2]. The many-body Kondo resonant tunneling was soon accounted for this phenomenon as an explanation [3]. The same effect which in low temperature (below the Kondo temperature $T_{\mathrm{K}}$ ) causes a resistance minimum in metal-diluted paramagnetic impurities, gives rise to a peak in conductance in the low-dimensional quantum dots (QD's) of various origin (such as two-dimensional electron gas (2DEG) [4, 5], carbon nanotubes [6], or molecules). It has been also observed in a single QD coupled to ferromagnetic leads [7-10], in which different coupling of opposite spin levels to electrodes, due to spin asymmetry of the density of states in ferromagnets, causes different levels' positions renormalization, which appears as a double-peak structure in the conductance-voltage characteristic [11-15]. The ZBA has not, however, been yet observed in magnetic tunnel junctions (MTJs) with paramagnetic impurities inside the tunnel barriers [16-18], although such experiment would answer some interesting questions. It has been widely believed that high magnetic moment systems cannot produce the Kondo effect that is overwhelmed by strong ferromagnetic interaction. We show that this belief appears not to be true and that ZBA anomaly does exist in planar MTJs with a CoFe nanodot layer coupled to ferromagnetic leads leading to the two-peak structure. The theoretical analysis of magnetic field and temperature dependences supports the interpretation of the experimental findings very well, showing and explaining distinct features of the conductance lines

* corresponding author; e-mail: eleyang@nus.edus.sg for the planar junctions with macroscopic number of interacting QD's.

\section{Experimental}

The junctions of area $S \approx 700 \mu \mathrm{m} \times 700 \mu \mathrm{m}$ were prepared using magnetron sputtering through a sequence of metal shadow masks at room temperature. The MTJs with a nanodot layer were formed from $100 \mathrm{Ta} / 250 \mathrm{Ir}_{22} \mathrm{Mn}_{78} / 35 \mathrm{Co}_{70} \mathrm{Fe}_{30} / 8 \mathrm{Mg} / 26$ $\mathrm{MgO} / 5 \quad \mathrm{Co}_{70} \mathrm{Fe}_{30} / 8 \mathrm{Mg} / 26 \quad \mathrm{MgO} / 70 \quad \mathrm{Co}_{70} \mathrm{Fe}_{30} / 150$ $\mathrm{Ir}_{22} \mathrm{Mn}_{78} / 50 \mathrm{Ta}$ (thicknesses in $\AA$ ). The $\mathrm{MgO}$ barriers were formed by reactive magnetron sputtering in an $\mathrm{Ar}-\mathrm{O}_{2}$ mixture [19]. Because the bottom electrode's exchange bias is stronger than that of the upper one, the magnetic moments of each electrode could be oriented independently. The $5 \AA$ layer of CoFe sputtered in the middle of the $\mathrm{MgO}$ layer plays a role of a nanodot-populated region (as shown in Fig. 1a) with the average diameter of the nanodots estimated to be $\varnothing \approx 2.6 \mathrm{~nm}$. Electron energy loss spectroscopy shows that the nanodots are in metallic state, and an assumption for them to be in paramagnetic state as well is reasonable [20]. Hundreds of shadow-masked MTJs were studied using a standard four-probe method and their temperature-dependent magneto-conductance characteristics were measured. We report the data from the most representative sample since all samples showed qualitatively similar behavior.

\section{Results}

In other typical tunneling Kondo experiments in tunnel junctions with normal metallic electrodes [1, 2, 4-6], a conductance peak was observed centered at zero bias voltage, whereas in our case of ferromagnetic electrodes at low bias a double-peak structure was observed shown in Fig. $1 \mathrm{~b}$ at $T=0.25 \mathrm{~K}$. In contrary to the expectation, 

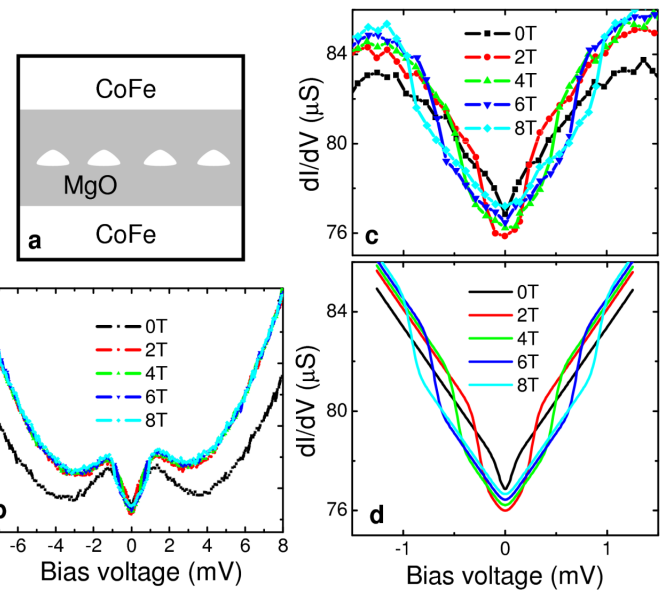

Fig. 1. (a) Schematic illustration of a cross-section of the MTJ with a discontinuous magnetic layer forming a layer of nanodots within the $\mathrm{MgO}$ layer. (b,c) Magnetic field dependence of the conductance as a function of the bias dependence at $T=0.25 \mathrm{~K}$ for a junction composed of $\mathrm{CoFe} / 26 \AA \mathrm{MgO} / 5 \AA \mathrm{CoFe} / 26 \AA \mathrm{MgO} / \mathrm{CoFe}$. (d) Theoretical fit to (c) using a second-order perturbation (cotunneling) theory with an additional residual field $B_{\text {res. }}=0.35 \mathrm{~T}$, an additional shift in the current by $+0.15 \mu \mathrm{S} / \mathrm{T}$, and strong spin-flip relaxation in the dots.

ferromagnetism suppresses the Kondo tunneling effect, a double peak in conductance [and a tunneling magnetoresistance (TMR) suppression as a consequencel appears at low bias voltages and temperatures. Such effects are similar to the previously observed Kondo resonance effect, appearing in $\mathrm{C}_{60}$ molecules as well as in carbon nanotubes, and semiconducting dots placed between ferromagnetic electrodes [7-9]. A calculation considering a $g$-factor, $g \approx 2$, shows that magnetic field leading $B$ to a splitting of $\Delta V=3 \mathrm{mV}$ would have to be as strong as $14 \mathrm{~T}$. It can be explained by the presence of the exchange interaction between a dot and ferromagnetic leads which was observed in the previous single dot experiments $[7,8]$. The spin asymmetry in the density of states in ferromagnetic electrodes produces a spin-dependent renormalization of dot's levels. The degeneracy is, thus, broken: $\epsilon_{\uparrow} \neq \epsilon_{\downarrow}$, and a spin splitting is observed, $\Delta \epsilon \equiv \epsilon_{\uparrow}-\epsilon_{\downarrow}$, rising a splitting of the conductance curve line similar to the one that can be caused by a strong enough magnetic field.

According to the theory for the Kondo effect the ZBA should fade and disappear with increasing temperature over the Kondo temperature $T_{\mathrm{K}}$. Our experiments show that that the observed double peak in conductance gradually evolves to a peak with a small splitting and the zero bias anomaly completely disappears above $T=15 \mathrm{~K}$, which defines $T_{\mathrm{K}}$ for this sample.

\section{Theoretical model}

One of the specific properties of spin-related effects, observed in our experiments is a dip broadening (inside of a double peak) when the magnetic field increases shown in Fig. 1b,c. We have, on contrary, not seen the effect in MTJs without a nanodot layer that also supports the fact that the Kondo effect happens in the presence of ferromagnetism in magnetic tunnel junctions. The positions of the conductance peaks' maxima are surprisingly almost unmodified by the magnetic field. The possible explanation of our observations is a superposition of the conductance of the Kondo quantum dots (with weak field dependence on magnetic field), strongly coupled to the leads and the conductance of the weaker coupled dots which are in the cotunneling regime.

We model the system with a Hamiltonian for a single level QD of energy $\epsilon_{\sigma}$ coupled to ferromagnetic leads

$$
\begin{aligned}
H & =\sum_{r k \sigma} \varepsilon_{r k \sigma} c_{r k \sigma}^{\dagger} c_{r k \sigma}+\sum_{\sigma} \epsilon_{\sigma} d_{\sigma}^{\dagger} d_{\sigma}+\frac{U}{2}(n-1 / 2)^{2} \\
& +\sum_{r k \sigma}\left(V_{r k} d_{\sigma}^{\dagger} c_{r k \sigma}+V_{r k}^{*} c_{r k \sigma}^{\dagger} d_{\sigma}\right)
\end{aligned}
$$

where $c_{r k \sigma}$ and $d_{\sigma}$ are the Fermi operators for electrons with wave vector $k$ and spin $\sigma$ in the leads, $r=\mathrm{L}, \mathrm{R}$, and in the QD, respectively. Here $V_{r k \sigma}$ is the spin-dependent tunneling amplitude, $n=\sum_{\sigma} d_{\sigma}^{\dagger} d_{\sigma}$ is the dot occupation, and $U$ is the on-site electron interaction. The difference $\epsilon_{\uparrow}-\epsilon_{\downarrow}=\Delta_{\mathrm{Z}}$ is induced by the Zeeman energy due to an external magnetic field, $\Delta_{\mathrm{Z}}=g \mu_{\mathrm{B}} B$. We discuss the cotunneling transport in the Coulomb-blockade valley in which dot's occupancy is fixed to one electron. The tunnel-coupling strength is characterized by $\Gamma_{r}^{\alpha}=2 \pi \rho_{r \sigma}\left|V_{r}\right|^{2}$, where $\rho_{r \sigma}$ denotes the density of states (for simplicity, we ignore $k$ dependence of $V_{r k}=V_{r}$ ). For ferromagnetic leads we consider the spin asymmetry defined in the standard way as $P \equiv\left(\rho_{r \uparrow}-\rho_{r \downarrow}\right) /\left(\rho_{r \uparrow}+\rho_{r \downarrow}\right)$.

The broadening of the dip in the conductance signal can be modeled by cotunneling — a simplest many-body process of the second order - in which only two electrons are involved. Using the second-order perturbation theory $[21,22]$ the tunneling rate $\gamma_{r r^{\prime}}^{\sigma, \sigma^{\prime}}$ is determined. It represents a process of an electron with spin state $\sigma$ entering the dot in state $\epsilon_{\sigma^{\prime}}$ from lead $r^{\prime}$ and leaving the dot state $\epsilon_{\sigma}$ when escaping to lead $r^{\prime}(r=\mathrm{L}, \mathrm{R}$ and $\sigma=\uparrow, \downarrow)$. Two modes of cotunneling can be defined here: elastic $\left(\sigma=\sigma^{\prime}\right)$ and inelastic spin-flip cotunneling $\left(\sigma \neq \sigma^{\prime}=\bar{\sigma}\right.$, where the dashed $\sigma$ means a spin opposite to $\sigma$ ) [23]. The first case of the elastic cotunneling is insensitive to spin splitting $\Delta \epsilon \equiv \epsilon_{\uparrow}-\epsilon_{\downarrow}$ but in the latter case, the current flows only if $|e V|>|\Delta \epsilon|$.

For $\sigma \neq \bar{\sigma}$, i.e., inelastic cotunneling, the rate is given by

$$
\begin{aligned}
& \gamma_{r r^{\prime}}^{\sigma, \bar{\sigma}}=\frac{\Gamma_{r}^{\sigma} \Gamma_{r^{\prime}}^{\bar{\sigma}}}{h} \mathrm{P} \int \mathrm{d} \omega \mathrm{d} \omega^{\prime} f(\omega)\left[1-f\left(\omega^{\prime}\right)\right] \\
& \quad \times\left[1 /\left(\omega^{\prime}+\mu_{r^{\prime}}-\epsilon_{\sigma}\right)+1 /\left(\epsilon_{\bar{\sigma}}+U-\omega-\mu_{r}\right)\right]^{2} \\
& \quad \times \delta\left(\omega+\mu_{r}-\omega^{\prime}+\mu_{r^{\prime}}+\epsilon_{\sigma}-\epsilon_{\bar{\sigma}}\right),
\end{aligned}
$$

where $f(\omega)$ denotes the Fermi function and $\mu_{r}$ is the electrochemical potential, and $\mathrm{P}$ denotes the principal value 
of integral. For elastic cotunneling $\sigma=\sigma^{\prime}$, when the dot state is not changed, we get

$$
\begin{aligned}
& \gamma_{r r^{\prime}}^{\sigma}=\frac{1}{h} \mathrm{P} \int \mathrm{d} \omega \mathrm{d} \omega^{\prime} f(\omega)\left[1-f\left(\omega^{\prime}\right)\right] \\
& \quad \times \delta\left(\omega+\mu_{r}-\omega^{\prime}+\mu_{r^{\prime}}\right) \\
& \quad \times\left[\frac{\Gamma_{r}^{\sigma} \Gamma_{r^{\prime}}^{\sigma}}{\left(\omega^{\prime}+\mu_{r^{\prime}}-\epsilon_{\sigma}\right)^{2}}+\frac{\Gamma_{r}^{\bar{\sigma}} \Gamma_{r^{\prime}}^{\bar{\sigma}}}{\left(\epsilon_{\bar{\sigma}}+U-\omega-\mu_{r}\right)^{2}}\right] .
\end{aligned}
$$

The current $I=I_{\mathrm{el}}+I_{\text {inel }}$ can be separated into the elastic $I_{\mathrm{el}}$ and inelastic $I_{\text {inel }}$ components

$$
\begin{aligned}
& I_{\mathrm{el}}=e \sum_{\sigma} p_{\sigma}\left(\gamma_{\mathrm{LR}}^{\sigma}-\gamma_{\mathrm{RL}}^{\sigma}\right), \\
& I_{\text {inel }}=e \sum_{\sigma \neq \bar{\sigma}} p_{\sigma}\left(\gamma_{\mathrm{LR}}^{\sigma \bar{\sigma}}-\gamma_{\mathrm{RL}}^{\sigma \bar{\sigma}}\right),
\end{aligned}
$$

where $p_{\sigma}$ is the probability of occupation of state $\sigma$. The probabilities $p_{\sigma}$ and $p_{\bar{\sigma}}$ are obtained from stationary rate equation $0=\sum_{r r^{\prime}}\left(p_{\sigma} \gamma_{r r^{\prime}}^{\sigma \bar{\sigma}}-p_{\bar{\sigma}} \gamma_{r r^{\prime}}^{\beta \bar{\sigma}}\right)$ together with the normalization condition $p_{\uparrow}+p_{\downarrow}=1$. In the weak coupling regime, when the current is weak, the intra-dot spin-flip relaxation becomes relevant. In this regime, the level occupation of the dot approaches the Boltzmann distribution [22] that can be obtained from the generalized rate equation $0=p_{\sigma} R\left(\epsilon_{\sigma}-\epsilon_{\bar{\sigma}}\right)-p_{\bar{\sigma}} R\left(\epsilon_{\bar{\sigma}}-\epsilon_{\sigma}\right)+$ $\sum_{r r^{\prime}}\left(p_{\sigma} \gamma_{r r^{\prime}}^{\sigma \bar{\sigma}}-p_{\bar{\sigma}} \gamma_{r r^{\prime}}^{\bar{\sigma} \sigma}\right)$, where the relaxation rate is given by $R(\omega)=R_{s f} \exp \left(-\omega / 2 k_{\mathrm{B}} T\right)$.

The case of the elastic cotunneling is insensitive to spin splitting $\Delta \epsilon \equiv \epsilon_{\uparrow}-\epsilon_{\downarrow}$ but in the inelastic case, the current flows only if $|e V|>|\Delta \epsilon|$. The theoretical results agree very well with the experimental findings, as it can be seen in Fig. 1c and d. In our calculations we use $P=0.5$ and find also that the best fit is obtained, when a strong spin-flip relaxation is considered, and a some additional residual field $B_{\text {res }}=0.35 \mathrm{~T}$ is added to the external magnetic field $B$.

We have demonstrated that planar macroscopic MTJs with magnetic nanodots can exhibit the ZBA such as a conductance double peak. Different coupling of electrons to the electrodes with opposite spins leads to a energy level splitting, similar to one caused by the magnetic field. That splitting can be observed as a double peak Kondo ZBA. Finally, the zero-bias tunneling anomalies are modified by the magnetic field and temperature according to the Anderson quantum dot model coupled to ferromagnetic electrodes which indicates the possibility of the coexistence of the Kondo effect and ferromagnetism in magnetic tunnel junctions.

\section{Acknowledgments}

We thank J. Barnaś, J. König, S. Maekawa, Yu.V. Nazarov, Y. Utsumi, I. Weymann, and G. Schön for helpful discussions. This work was supported by the Singapore Ministry of Education Academic Research
Fund Tier 2 (MOE2008-T2-1-105) and by a Polish grant for science for the years 2010-2013.

\section{References}

[1] A.F.G. Wyatt, Phys. Rev. Lett. 13, 401 (1964).

[2] D.J. Lythall, A.F.G. Wyatt, Phys. Rev. Lett. 20, 1361 (1968).

[3] A.C. Hewson, The Kondo Problem to Heavy Fermions, Cambridge Univ. Press, Cambridge 1993.

[4] D. Goldhaber-Gordon, Hadas Shtrikman, D. Mahalu, David Abusch-Magder, U. Meirav, M.A. Kastner, $\mathrm{Na}$ ture 391, 156 (1998).

[5] S.M. Cronenwett, T.H. Oosterkamp, L.P. Kouwenhoven, Science 281, 540 (1998).

[6] J. Nygard, D.H. Cobden, P.E. Lindelof, Nature 408, $342(2000)$.

[7] A.N. Pasupathy, Science 306, 86 (2004).

[8] J.R. Hauptmann, J. Paaske, P.E. Lindelof, Nature Phys. 4, 373 (2008).

[9] K. Hamaya, M. Kitabatake, K. Shibata, M. Jung, M. Kawamura, K. Hirakawa, T. Machida, T. Taniyama, S. Ishida, Y. Arakawa, Appl. Phys. Lett. 91, 232105 (2007).

[10] M.R. Calvo, J. Fernández-Rossier, J.J. Palacios, D. Jacob, D. Natelson, C. Untiedt, Nature 458, 1150 (2009).

[11] J. Martinek, Y. Utsumi, H. Imamura, J. Barnaś, S. Maekawa, J. König, G. Schön, Phys. Rev. Lett. 91, 127203 (2003).

[12] J. Martinek, J. Barnaś, A. Fert, S. Maekawa, G. Schön, J. Appl. Phys. 90, 8265 (2003).

[13] J. Martinek, Y. Utsumi, H. Imamura, J. Barnaś, S. Maekawa, J. König, G. Schön, Phys. Rev. Lett. 91, 247202 (2003).

[14] M.-S. Choi, D. Sanchez, R. Lopez, Phys. Rev. Lett. 92, 056601 (2004).

[15] Y. Utsumi, J. Martinek, G. Schön, H. Imamura, S. Maekawa, Phys. Rev. B 71, 245116 (2005).

[16] L.F. Schelp, A. Fert, F. Fettar, P. Holody, S.F. Lee, J.L. Maurice, F. Petroff, A. Vaurès, Phys. Rev. B 56, R5747 (1997).

[17] H. Sukegawa, S. Nakamura, A. Hirohata, N. Tezuka, K. Inomata, Phys. Rev. Lett. 94, 068304 (2005).

[18] K. Yakushiji, F. Ernult, H. Imamura, K. Yamane, S. Mitani, K. Takanashi, S. Takahashi, S. Maekawa, H. Fujimori, Nature Mater. 4, 57 (2005).

[19] S.S.P. Parkin, C. Kaiser, A. Panchula, P.M. Rice, B. Hughes, M. Samant, S.-H. Yang, Nature Mater. 3, 862 (2004).

[20] H. Yang, S. Yang, S. Parkin, Nano Lett. 8, 340 (2008).

[21] I. Weymann, J. Barnaś, J. König, J. Martinek, G. Schön, Phys. Rev. B 72, 113301 (2005); 72, 115334 (2005).

[22] S. Moriyama, J. Martinek, G. Ilnicki, T. Fuse, K. Ishibashi, Phys. Rev. B 80, 033408 (2009).

[23] A.J. Heinrich, J.A. Gupta, C.P. Lutz, D.M. Eigler, Science 306, 466 (2004). 